\section{PTU-113 $\star$ RECTAL MUCOSAL BIOMARKERS OF COLORECTAL CANCER RISK ARE INCREASED IN MORBIDLY OBESE PATIENTS BUT NOT SIGNIFICANTLY DIFFERENT SIX MONTHS AFTER SLEEVE GASTRECTOMY}

doi:10.1136/gut.2011.239301.241

P Kant, ${ }^{1, *}$ S Dexter, ${ }^{2}$ M A Hull1 ${ }^{1}$ Section of Molecular Gastroenterology, Institute of Molecular Medicine, University of Leeds, Leeds, UK; ${ }^{2}$ Upper Gastrointestinal Surgery, St James' University Hospital, Leeds, UK

Introduction We have previously shown that putative mucosal biomarkers of colorectal cancer (CRC) risk (rectal epithelial cell mitosis frequency and crypt size) are significantly higher in morbidly obese (body mass index (BMI) $>40 \mathrm{~kg} / \mathrm{m}^{2}$ ) patients than normal weight controls. These biomarkers were further increased up to 3 years after Roux-en-Y gastric bypass (RYGB), despite significant weight loss. We tested whether CRC risk biomarkers were similarly affected by sleeve gastrectomy (SG), a form of bariatric surgery not associated with malabsorption secondary to intestinal bypass.

Methods Serum and rectal mucosa were obtained from 18 morbidly obese patients (mean BMI $64.1 \mathrm{~kg} / \mathrm{m}^{2}$ ) before and 6 months after SG, as well as from 18 non-obese (BMI 24.7 $\mathrm{kg} / \mathrm{m}^{2}$ ) patients undergoing anal canal surgery. Mucosal biomarkers were measured after whole crypt micro-dissection. Mucosal pro-inflammatory gene expression was measured by real-time PCR. Serum C-reactive protein (CRP), tumour necrosis factor (TNF)- $\alpha$ and interleukin (IL)- 6 levels were measured by immunoassay.

Results Before SG, morbidly obese patients had a higher epithelial cell mitosis frequency (mean 4.8 cells per crypt) compared with non-obese controls ( 3.3 cells per crypt, $p<0.05$ ), with a luminal shift in the distribution of mitotic cells. Morbidly obese patients had larger rectal crypts (mean area 0.039 vs $0.033 \mathrm{~mm}^{2}$; $\mathrm{p}<0.01)$ than non-obese individuals. SG was associated with a mean excess weight loss (EWL) of $38.6 \%$ at 6 months (similar to the mean EWL after RYGB). Following SG, serum CRP decreased from a median level of $17.5 \mathrm{mg} / \mathrm{l}$ to $9.9 \mathrm{mg} / \mathrm{l}(\mathrm{p}<0.001)$, but there was no significant change in serum IL-6 or TNF $\alpha$ levels. Unlike after RYGB, there was no significant change in epithelial cell mitosis frequency or crypt size 6 months after SG. The only mucosal pro-inflammatory gene transcript to significantly increase 6 months after SG (1.5-fold, 95\% CI 1.1 to 2.1, $\mathrm{p}<0.05$ ) was macrophage migration inhibitory factor (MIF).

Conclusion We have confirmed that mucosal CRC risk biomarkers are elevated in morbidly obese patients compared with non-obese individuals. Significant SG-induced weight loss was not associated with lower CRC risk biomarker values. Unlike RYGB, SG was not associated with a significant increase in epithelial cell mitosis frequency or crypt size 6 months after surgery. However, SG was associated with increased mucosal expression of the pro-tumourigenic cytokine MIF, consistent with our previous observations in RYGB patients. Post-surgical mucosal and inflammatory biomarkers vary in morbidly obese patients depending on the type of bariatric surgery undertaken. Prospective studies of colorectal neoplastic risk in obese patients after bariatric surgery are warranted.

Competing interests None.

Keywords colorectal cancer, obesity. 